\title{
Design of a low-noise aeroacoustic wind tunnel facility at Brunel University
}

\author{
Alexandros Vathylakis ${ }^{1}$, Tze Pei Chong ${ }^{2}$ and Jung Hoon Kim ${ }^{3}$ \\ School of Engineering and Design, Brunel University, Uxbridge, UB8 3PH, UK
}

\begin{abstract}
This paper represents the design principle of a quiet, low turbulence and moderately high speed aeroacoustic wind tunnel which was recently commissioned at Brunel University. A new hemi-anechoic chamber was purposely built to facilitate aeroacoustic measurements. The wind tunnel can achieve a maximum speed of about $80 \mathrm{~ms}^{-1}$. The turbulence intensity of the free jet in the potential core is between $0.1-0.2 \%$. The noise characteristic of the aeroacoustic wind tunnel was validated by three case studies. All of which can demonstrate a very low background noise produced by the bare jet in comparison to the noise radiated from the cylinder rod/flat plate/airfoil in the air stream.
\end{abstract}

\section{Nomenclature}

$2 h=$ serration amplitude (root-to-tip distance) of the sawtooth

$A \quad=$ aperture of the screen

$A_{\text {inlet }}=$ inlet duct area immediately after the centrifugal fan

$A_{\text {outlet }}=$ outlet area of the nozzle exit inside the anechoic chamber

$A R=$ area ratio

$B \quad=$ porosity of the screen

$C=$ airfoil chord length

$C R=$ contraction ratio of the nozzle

$D \quad=$ diameter of the screen wire

$f \quad=$ frequency

$K=$ pressure drop coefficient of the screen

$P^{\prime} \quad=$ acoustic pressure fluctuations

$P \quad=$ pitch of the screen

$N_{1}, N_{2}=$ number of measurement points correspond to $A_{\text {inlet }}$ and $A_{\text {outlet }}$, respectively

$T L=$ transmission loss of the silencer

$x, y, z=$ coordinates in the streamwise, vertical and spanwise directions, respectively

$\varphi \quad=$ serration angle of the sawtooth

\section{Introduction}

$\mathrm{T}$ he difference between an aerodynamic wind tunnel and an aeroacoustic wind tunnel is normally not large, except that the latter type tends to produce air stream which has a low-noise characteristic. If a test object is submerged in the quiet air stream within a sound diffusing environment, aero-acoustic radiation from the test object and the spectral information could then be quantified with a reasonable accuracy.

Aeroacoustic wind tunnels are continuously designed and installed by university research groups around the world. A very well known aeroacoustic wind tunnels is in the Virginia Tech of the United States ${ }^{1}$. The $1.83 \mathrm{~m} \times 1.83$

\footnotetext{
${ }^{1} \mathrm{PhD}$ student, School of Engineering and Design, alexandros.vathylakis@brunel.ac.uk, AIAA Student Member

${ }^{2}$ Lecturer, School of Engineering and Design, t.p.chong@brunel.ac.uk, AIAA Member

${ }^{3}$ Post-doctoral research fellow, School of Engineering and Design, junghoon.kim@brunel.ac.uk, AIAA Member 
$\mathrm{m}$ test section can achieve maximum speed of about $75 \mathrm{~ms}^{-1}$ without blockage. The test section, which is inside an anechoic chamber, consists of two Kevlar-clothed side walls. These side walls act like a normal flow surface, but they are also acoustically "transparent". For a high pressure loading experiment, both the aerodynamically and acoustically free field conditions can be achieved. A slightly smaller anechoic wind tunnel facility at University of Florida ${ }^{2}$ consists of a test section of $0.74 \mathrm{~m} \times 1.12 \mathrm{~m}$. The air jet produced by the open nozzle will expand and a downstream diffuser will collect the air. Both the open nozzle and the diffuser are situated inside an anechoic chamber. A fan is placed downstream of the diffuser to create pressure difference and air is sucked through the upstream nozzle. The maximum test section speed is reported as $76 \mathrm{~ms}^{-1}$. Background noise level produced by the free jet is shown to be very low.

Many university-based aeroacoustic wind tunnels can also be found in Europe. The open jet wind tunnel anechoic facility at the Ecole Centrale de Lyon has already been extensively used for experimental airfoil noise studies $^{3,4}$. Few years ago, a quiet and low turbulence open jet wind tunnel (DARP Rig) was installed at the Institute of Sound and Vibration Research, University of Southampton ${ }^{5}$. The jet speed can achieve more than $100 \mathrm{~ms}^{-1}$ through a $150 \mathrm{~mm} \times 450 \mathrm{~mm}$ nozzle. At the same time, extremely low background noise is emitted, which enables the aeroacoustic measurements on the airfoil noise and car window noise to be carried out there ${ }^{6-9}$. The key performance targets of the DAPR rig are to produce low noise and low turbulence free jet, which are achieved by two large silencers, extensive woven wire mesh screens throughout the circuit and a carefully-designed nozzle. More recently, the Brandenburg Technical University Cottbus in Germany commissioned a new low noise wind tunnel ${ }^{10}$ and it is suitable to be used in both the reverberant and anechoic environments. Circular and rectangular nozzles of different contraction ratios can be interchanged, which produce approximately $0.2 \%$ and $0.1 \%$ freestream turbulence intensities, respectively. Many airfoil self noise studies have already been performed in this facility ${ }^{11,12}$. The University of Adelaide from Australia built an aeroacoustic wind tunnel facility which is situated in an $8 \mathrm{~m}^{3}$ cubic anechoic chamber with a cut-off frequency at $200 \mathrm{~Hz}^{13}$. A nozzle-diffuser configuration is adopted, where a centrifugal fan is used to propel air through a silencer, settling drum and nozzle before entering the collecting diffuser. The maximum speed of the free jet is reported to be around $40 \mathrm{~ms}^{-1}$ through a $225 \mathrm{~mm} \times 75 \mathrm{~mm}$ nozzle exit. Similarly, low background noise is reported and many airfoil self noise studies have been carried out using this facility $^{14,15}$. There are also other university-based aeroacoustic wind tunnels, for example in refs ${ }^{16-18}$, which have been built for different purposes.

A brand new aeroacoustic research facility is recently commissioned at Brunel University in the United Kingdom. This facility is intended for airfoil noise study mainly in the low-to-moderate pressure loading configurations initially. A new $4 \mathrm{~m} \times 5 \mathrm{~m} \times 3.4 \mathrm{~m}$ hemi-anechoic chamber is also purposely built to house the aeroacoustic wind tunnel and to facilitate the far field noise measurements. The main objective of this paper is to present the design principle of the aeroacoustic wind tunnel (Sections II and III). Freestream turbulence intensity of the exit jet is measured and presented in Section IV. The acoustical performance of the wind tunnel is validated by three case studies in Section V. The first case concerns the tonal noise produced by the flow over a cylinder rod with rough surface. The second case concerns a flat plate flush mounted to one side of the nozzle exit. The boundary layer was tripped near the nozzle exit and noise measurements were performed for the flat plate with and without a serrated sawtooth trailing edge. The third case investigates the broadband self noise generated by a tripped NACA0012 airfoil at zero angle of attack. The conclusion and outlook are discussed in Section VI.

\section{Design considerations}

The open jet wind tunnel at Brunel University aims to achieve low noise radiation and low residual turbulence in the free jet. The major design constraint is the rather restricted $5.8 \mathrm{~m}$ (width) $\times 9.0 \mathrm{~m}$ (length) $\times 4.6 \mathrm{~m}$ (height) available room space which should accommodate every component of the open jet wind tunnel, including the centrifugal fan, as well as the anechoic chamber. The initial concern was that noise generated by the centrifugal fan will propagate with the flow inside the air duct. A silencer with a high transmission loss is used to attenuate the airborne noise. Another concern is that the centrifugal fan noise can penetrate through the anechoic chamber wall directly. To minimize such impact an acoustically lined-compartment could be built to "contain" the centrifugal fan.

It was decided that the open jet wind tunnel should be the blower type capable of producing a maximum mass flow rate of about $3.0 \mathrm{kgs}^{-1}$. A nozzle contraction ratio, $C R$, of $20-25$ is pre-selected to achieve low turbulence intensity for the free jet, which is set at below $0.3 \%$ as the performance target. In order to minimize flow separation and excessive pressure drop within the wind tunnel circuit, the change of cross-sectional area in the axial direction will be minimized. For the case when there is a curve duct, the area ratio, $A R$, will be kept close to unity. To achieve a low background noise of the free jet, a large in-line silencer will be installed. The acoustical performance target is 


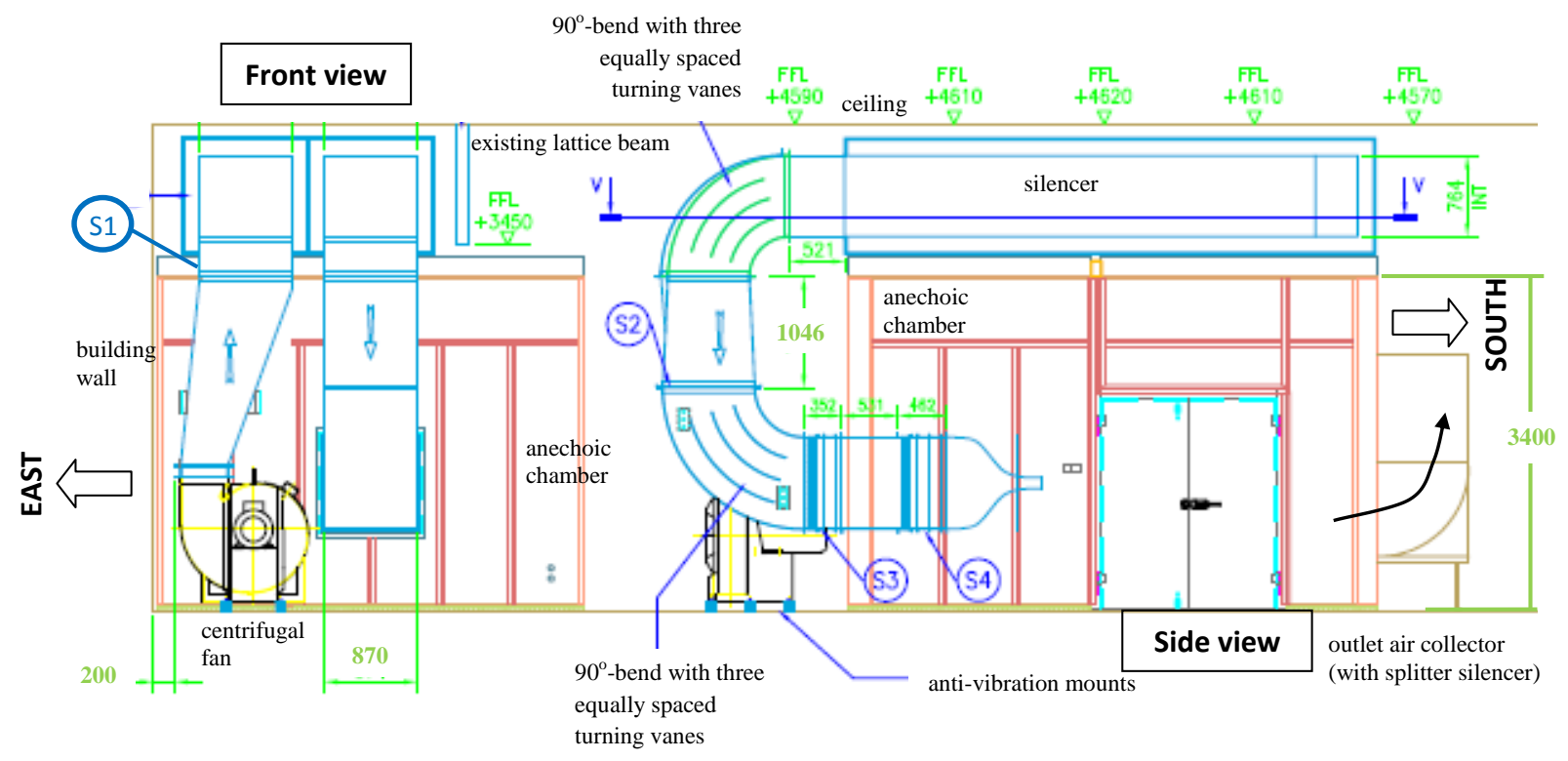

Figure 1. Plan, side and front views of the aeroacoustic wind tunnel and the anechoic chamber.

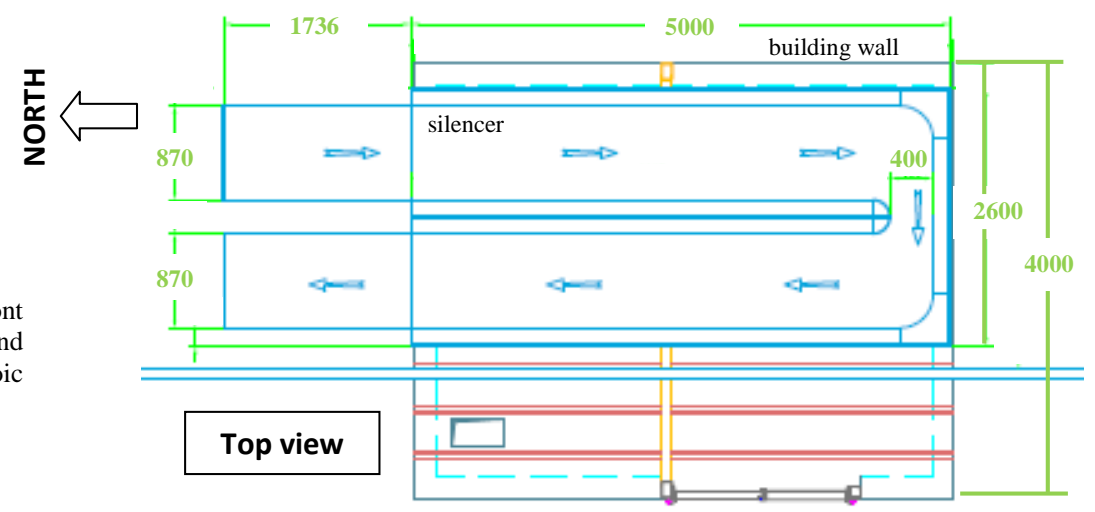

decided upon by the background noise to be at least $5 \mathrm{~dB}$ lower than the airfoil/flat plate trailing edge broadband self noise at a free jet velocity of at least $80 \mathrm{~ms}^{-1}$. The overall layout of the open jet wind tunnel with respect to the anechoic chamber, as well as some of the design details for each wind tunnel components, will be presented in the next section.

\section{The open jet wind tunnel}

During the previous experience in designing the Southampton DARP rig, the open jet wind tunnel has to be adapted to the existing ISVR anechoic chamber and the surrounding building structures. For example, the short, large area ratio and $90^{\circ}$-curved diffuser represents a supercritical and unfavorable part of the wind tunnel in order to overcome some very tight spaces ${ }^{19}$. Although the rather small room space available for the aeroacoustic wind tunnel at Brunel represents a design constraint, there is also an advantage that each components of the wind tunnel, including the anechoic chamber, can be treated as the "design variable". After several design iterations, the overall dimension of the anechoic chamber is decided to be $4 \mathrm{~m}$ (width) $\times 5 \mathrm{~m}$ (length) $\times 3.4 \mathrm{~m}$ (height). As shown in Fig. 1, the anechoic chamber is placed against the east side of the wall, leaving clearances of $2.0 \mathrm{~m}, 1.8 \mathrm{~m}$ and $1.8 \mathrm{~m}$ from the west, north and south sides of the walls, respectively. The wind tunnel enters the anechoic chamber from the north side, whereas a $90^{\circ}$-bend acoustically-lined outlet attenuator (air collector) is placed at the south side of the anechoic chamber for air ventilation purpose.

The plan, side and front views of the aeroacoustic wind tunnel are shown in Fig. 1. A centrifugal fan is placed at the north side to propel air vertically upward through an offset diffuser. The expanded air is then turned towards a $90^{\circ}$-bend duct $(A R=1)$ before enters the silencer, which is placed on top of the anechoic chamber. The air inside the silencer is flowing towards the south side, before turning $180^{\circ}$ at the far end and continuing its journey in the opposite direction towards the north side. After reaching the north end, the air exits the silencer and is turned by a $90^{\circ}$-bend duct $(A R=1)$ towards the floor. From there, the air is expanded a little bit before it is turned again towards 
$90^{\circ}$ anti-clockwise with a constant area duct and enters the anechoic chamber from the north side. After passing a series of flow conditioning devices, the air accelerates inside the nozzle and discharging to the atmosphere. The expanded jet will then reaches the acoustically lined-outlet attenuator and turns upwards through a splitter-type silencer, exiting the anechoic chamber.

Detailed descriptions of the major components of the aeroacoustic wind tunnel are provided below:

\section{A. Centrifugal fan}

In order to generate a maximum mass flow rate of $3.0 \mathrm{kgs}^{-1}$, the pressure rise which the fan should generate, after taking into account of the cumulative static pressure loss of the wind tunnel components, is slightly above $8 \mathrm{kPa}$. A $30 \mathrm{~kW}$ AC-powered centrifugal fan is chosen to fulfill the above requirements. An electric inverter is used to digitally-adjust the current input to the centrifugal fan, thus controlling the mass flow rate (the exit jet velocity) of the nozzle in an accurate manner.

As shown in Fig. 1, the centrifugal fan has to be placed quite close to the north side of the anechoic chamber. To minimize the structural noise transmission, an acoustically-lined compartment could be used to contain the centrifugal fan. The design of the compartment will take into considerations that it should not restrict the supply of fresh air to the fan's inlet and overheat the fan. This compartment has not been implemented yet. This is because a preliminary test did not reveal significant break-in of the centrifugal fan noise into the anechoic chamber. Nevertheless, there is a provision to add this feature to the wind tunnel in the future.

Another issue with the centrifugal fan is the possible structural vibration when in operation. In order to avoid the structurally-borne noise that could be transmitted via the floor, four anti-vibration mounts were used to isolate the centrifugal fan from the floor. Another possible route of transmission of the vibration is through the connection between the centrifugal fan and the wind tunnel components. To minimize this effect, a flexible vinyl tube is used to connect the centrifugal fan to the wind tunnel duct. This flexible tube is shown to be effective in dampening the vibration.

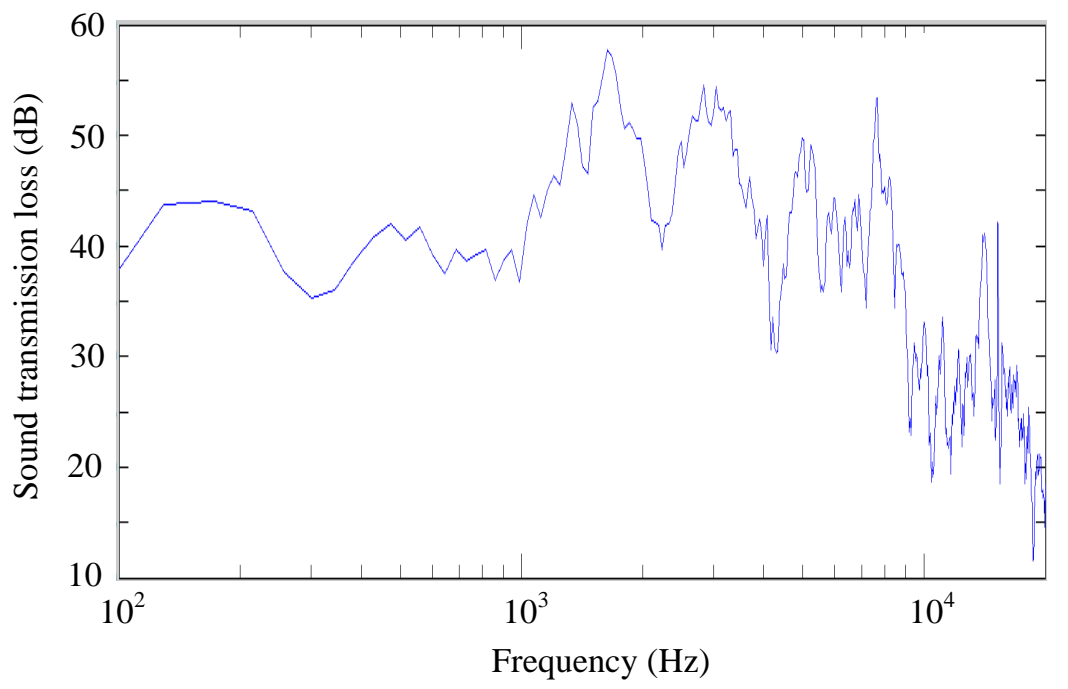

Figure 2. Measured sound transmission loss of the silencer

\section{B. Primary silencer}

A significant by-product of the centrifugal fan when in operation is the noise it produces alongside the air stream. If not treated properly, the noise component will propagate through the wind tunnel ducts and eventually radiate to the inside of the anechoic chamber. It is therefore important to attenuate the flow-borne noise as soon as possible. A $10 \mathrm{~m}$ long silencer is built for this purpose. The silencer is in the form of "2-pass" plenum chamber, which incorporates an $180^{\circ}$-lined bend. The cross-sectional area for each pass of the plenum chamber is about $1.2 \mathrm{~m}$ $\mathrm{x} 1.2 \mathrm{~m}$ (including the basalt wool liners and the plywood wall). All interior surfaces are lined with the basalt wool dissipative liner of $150 \mathrm{~mm}$ thickness with a $4 \mathrm{~mm}$ thick facing cloth (Thermal 650 E-glass Needlemat) to inhibit the 
flow delamination. The basalt wool liners are held together by the perforated metal frames. The outer wall of the silencer is made from the $18 \mathrm{~mm}$ thick plywood.

The choice of the acoustic material is based on the objective of achieving low flow resistivity and high density. A typical mineral wool used for the silencer, for example the Rockwool, produces flow resistivity between 20-40 $\mathrm{kPa} . \mathrm{s}^{-2}$ for a density range of $50-80 \mathrm{kgm}^{-3}$, respectively $\mathrm{y}^{20}$. However, a basalt wool could produce a flow resistivity of $15 \mathrm{kPa} . \mathrm{s}^{-2}$ at a density of $120 \mathrm{kgm}^{-3}$, which implies that a dense acoustic material could be used in a silencer whilst a superior sound absorbing performance is still maintained.

The acoustic transmission loss, $T L$, could be estimated in situ from the following relationship:

$$
T L=\frac{\sum_{i=1}^{N_{1}} P_{i}^{\prime}(f)_{\text {inlet }}}{\sum_{i=1}^{N_{2}} P_{i}^{\prime}(f)_{\text {outlet }}} \cdot \frac{N_{2}}{N_{1}} \cdot \frac{A_{\text {inlet }}}{A_{\text {outlet }}}
$$

Under a no flow condition, the noise source is emitted by a $100 \mathrm{~W}$ loudspeaker. The input signal to the loudspeaker is driven by a white noise generator and an audio amplifier. The loudspeaker is placed directly above the centrifugal fan ducted outlet with a cross-sectional area of $A_{\text {inlet }}$. A condenser microphone is used to measure the fluctuating sound pressures $P^{\prime}(f)_{\text {inlet }}$ at $N_{1}$ number of grid points within the $A_{\text {inlet }}$ to obtain the averaged acoustic spectrum. Similarly, the fluctuating sound pressures at the nozzle exit $P^{\prime}(f)_{\text {outlet }}$ are measured in $N_{2}$ number of grid points within the exit area of $A_{\text {outlet }}$ to obtain the averaged value. Figure 2 shows that between $40-55 \mathrm{~dB}$ transmission loss in the frequency range of $400-8000 \mathrm{~Hz}$ could be expected from this primary silencer. It is useful to point out that extra splitter type silencer could be added at a later date if more sound reduction is necessary. According to the layout in Fig. 1, the ideal places for the additional splitter silencer could either be at the diffuser upstream of the S2, or at the settling chamber between $\mathrm{S} 3$ and $\mathrm{S} 4$.

\begin{tabular}{|c|c|c|c|c|c|c|c|c|c|c|c|c|c|c|c|c|c|}
\hline \multirow[t]{2}{*}{ REF } & \multicolumn{2}{|c|}{ Honeycomb } & \multicolumn{5}{|c|}{ Woven Mesh Layer 1} & \multicolumn{5}{|c|}{ Woven Mesh Layer $\mathbf{2}$} & \multicolumn{5}{|c|}{ Woven Mesh Layer 3} \\
\hline & Cell dia. $(\mathrm{mm})$ & Thickness (mm) & $\mathrm{D}(\mathrm{mm})$ & $A(\mathrm{~mm})$ & $\mathrm{P}(\mathrm{mm})$ & B & $\mathrm{K}$ & $\mathrm{D}(\mathrm{mm})$ & $\mathrm{A}(\mathrm{mm})$ & $\mathrm{P}(\mathrm{mm})$ & $B$ & K & $\mathrm{D}(\mathrm{mm})$ & $A(\mathrm{~mm})$ & $\mathrm{P}(\mathrm{mm})$ & $\mathrm{B}$ & $\mathrm{K}$ \\
\hline S1 & & & 0.45 & 0.82 & 1.270 & 0.417 & 2.47 & & & & & & & & & & \\
\hline S2 & & & 0.28 & 0.57 & 0.850 & 0.450 & 2.05 & & & & & & & & & & \\
\hline S3 & 6.35 & 63.5 & 0.28 & 0.57 & 0.850 & 0.450 & 2.05 & 0.355 & 1 & 1.355 & 0.545 & 1.23 & & & & & \\
\hline S4 & 6.35 & 63.5 & 0.355 & 1 & 1.355 & 0.545 & 1.23 & 0.165 & 0.68 & 0.845 & 0.648 & 0.72 & 0.165 & 0.68 & 0.845 & 0.648 & 0.72 \\
\hline
\end{tabular}

$B=$ porosity

$K=$ pressure drop coefficient

$D=$ wire diameter

$A=$ aperture

$B=\left(1-\frac{D}{P}\right)^{2}$

$P=$ pitch

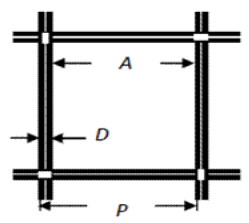

Table 1. Data for the honeycombs and screens used in the aeroacoustic wind tunnel. Refer to Fig. 1 for locations of the S1, S2, S3 and S4.

\section{C. $\mathbf{9 0}^{\circ}$-bend ducts and other flow conditioning devices}

As shown in Fig. 1, the wind tunnel consists of three $90^{\circ}$-bend ducts, all of which are with $A R=1$. Each bend also consists of three equally-spaced flow splitter vanes to guide the flow and to reduce the magnitude of the secondary flow due to the centrifugal force acting on the flow. Neither the large scale boundary layer separation, nor the flow unsteadiness, is expected to occur in these bend ducts.

The flow conditioning devices, as indicated in Fig. 1 for the S1, S2, S3 and S4 at different locations, mainly consist of woven wire screens and honeycombs. For the honeycomb, the chosen configuration fulfills the recommendation that the depth of the honeycomb should be greater than 10 times the cell diameter to allow adequate flow straightening $^{21}$. For the woven wire screen, a greater margin of pressure drop per screen is in favor to achieve a uniform flow. The pressure drop coefficient, $K$, generally works against the porosity square, $B^{2}$. Therefore, a screen with a smaller porosity will produce a high pressure drop. However, a lower limit on the porosity should be observed because the resulting configuration may result in jet coalescence between the meshes, which will add instabilities to the flow and increase the turbulence level. This means that there should be an upper limit on the 
pressure drop coefficient for each screen, which is recommended to be below 2.5 [ref 22]. Therefore, a common practice is to use several screens of low $K$-value instead of a single screen of large $K$-value, although the former case will inevitably increase the overall length of the "screen pack". Another design valuable, which associates with the screen porosity and the pressure drop coefficient, is the woven wire diameter, $D$. A mesh wire with a large $D$ can produce a cascade of turbulent eddies across the spectrum. Ideally, the wire diameter $D$ should be as small as possible to avoid excessive vortex shedding into the flow. The decay rate of the turbulence intensity generated by the vortex shedding from a cylindrical body is $\sim(x / D)^{-5 / 7}$. This dictates the minimum separation distance which must be considered between each screen. All of the design criteria described above have been taken into consideration. The final geometrical parameters of the honeycombs and woven wire screens for S1-S4 are reflected in Table 1.

\section{Nozzle}

A nozzle is used to convert the static pressure into the kinetic energy and to discharge the high speed air at the nozzle exit. The conversion process represents the key mechanism of straining the freestream turbulence eddies, thereby reducing the velocity fluctuations. Using a three-dimensional nozzle, the transverse and spanwise fluctuating components of the turbulence eddies could be reduced. The level of the eddies-straining is therefore a function of the area ratio $A R$ of the nozzle.

However, nozzle with a large area ratio and a comparatively small axial length will have a higher risk of boundary layer separation at the inflection point. On the other hand, nozzle with a longer axial length will be less prone to boundary layer separation, but it may also result in excessive growth of the boundary layer thickness near the nozzle exit. A benchmark nozzle similar to the DARP rig at Southampton $(A R=25)$ is manufactured and installed in the Brunel wind tunnel. The scaling factor between the DARP nozzle and the Brunel nozzle is $2 / 3$, which results in the following dimensions for the Brunel nozzle: inlet $=867 \mathrm{~mm} \times 867 \mathrm{~mm}$; outlet $=100 \mathrm{~mm} \times 300 \mathrm{~mm}$. For a mass flow rate of $3.0 \mathrm{kgs}^{-1}$, this represents a maximum free jet velocity of approximately $80 \mathrm{~ms}^{-1}$.

It is worth mentioning that the rather short nozzle height at the exit $(100 \mathrm{~mm})$ may not be suitable for airfoil experiment in the off-design, high pressure loading configurations due to the large jet deflection. There is a plan to manufacture another nozzle with a larger exit area in the future. To achieve a freeflow-like condition when the airfoil is set at a moderately large angle of attack, a method similar to the Virginia Tech's will be employed to connect the nozzle with a Kevlar-walled test section.

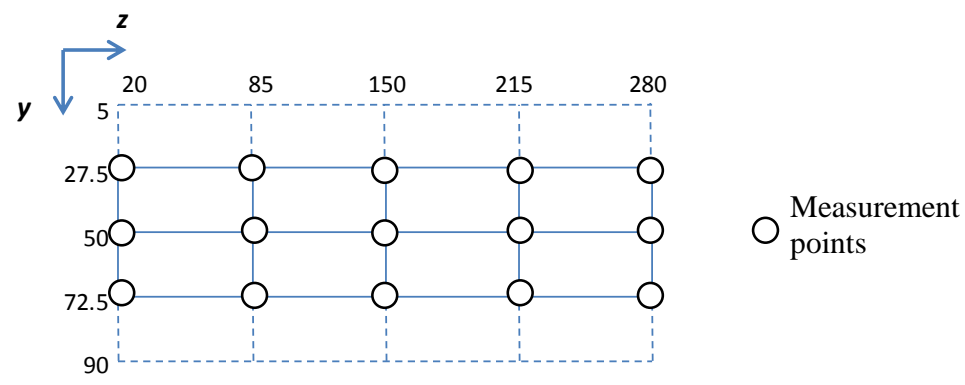

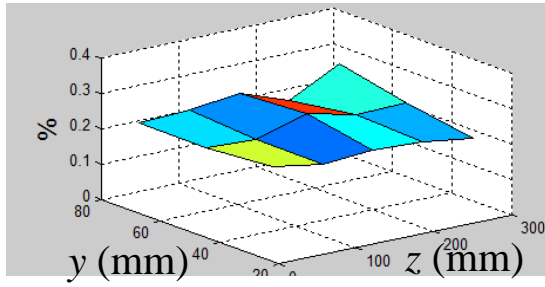

(a)

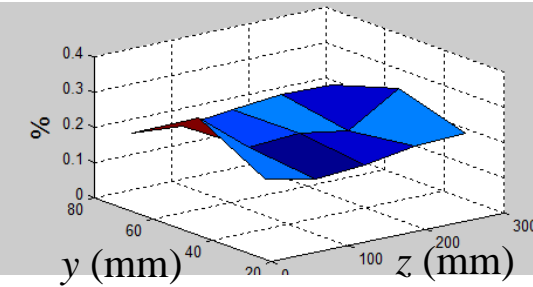

(b)

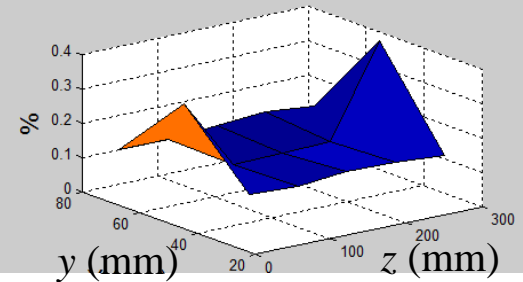

(c)

Figure 3. Distributions of the turbulence intensities at $8 \mathrm{~mm}$ downstream of the nozzle exit plane at jet velocity of (a). $10 \mathrm{~ms}^{-1}$, (b). $20 \mathrm{~ms}^{-1}$, and (c). $30 \mathrm{~ms}^{-1}$. 


\section{Turbulence intensities produced by the free jet}

The turbulence intensity of the free jet was measured with a single hot wire probe of $5 \mu \mathrm{m}$ diameter, and 1.25 $\mathrm{mm}$ wire length. Figure 3 shows the distributions of the turbulence intensities at 15 measurement points equally distributed at $8 \mathrm{~mm}$ downstream of the nozzle exit plane in the $y-z$ direction. The speed range was set at $10-30 \mathrm{~ms}^{-1}$ at an interval of $10 \mathrm{~ms}^{-1}$. From the figure, the potential core of the free jet has a typical range of turbulence intensity between $0.1-0.2 \%$, with the higher limit tends toward the lower end of the jet velocity. The results suggest that the initial target of below $3 \%$ turbulence intensity has been achieved, which would allow airfoil trailing edge noise to be the dominant noise source in an aerodynamically clean air stream.

\section{Acoustical performances of the open jet wind tunnel}

In this paper, instead of presenting the spectrum of the background noise in a bare jet configuration per se, the acoustical performance of the open jet wind tunnel will be assessed against several cases where test objects are placed in the air stream. The results are discussed in the following sections.

(a)

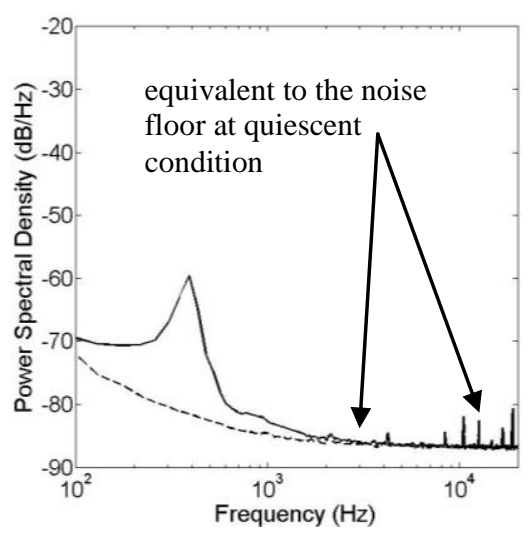

(b)

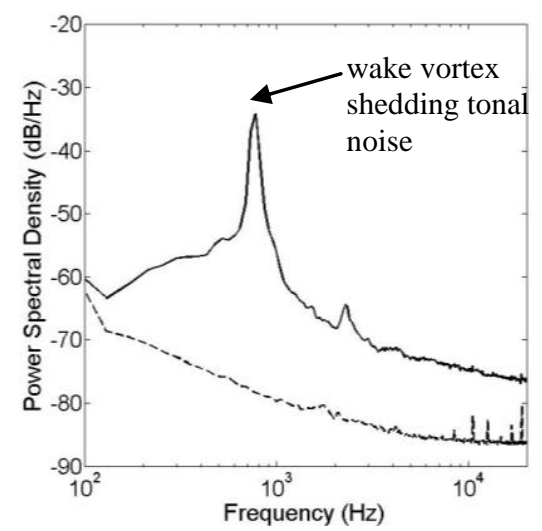

(c)

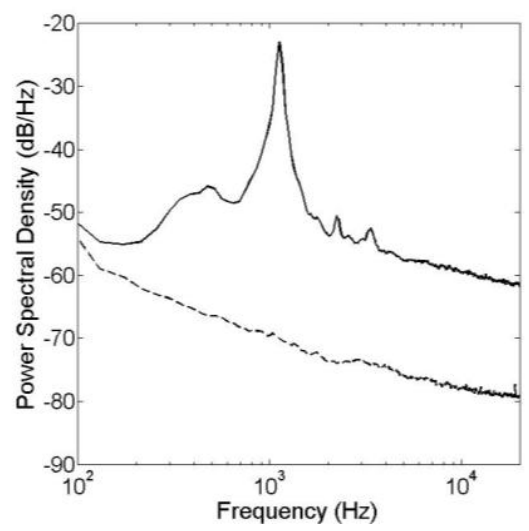

Figure 4. Comparisons of the noise radiated by the $12 \mathrm{~mm}$ diameter cylinder rod with rough surface (-) and the wind tunnel background noise produced by the bare jet (- -) at freestream velocities of: (a). $20 \mathrm{~ms}^{-1}$, (b). $40 \mathrm{~ms}^{-1}$ and (c). $60 \mathrm{~ms}^{-1}$.

\section{A. Vortex shedding noise from a bluff body}

In this test, a $12-\mathrm{mm}$ diameter rough surface cylinder rod was placed in the free jet at different speeds. This particular configuration is chosen due to the likelihood of a high amplitude tone and a moderate level of broadband noise to be radiated from the cylinder rod with rough surface. A condenser microphone was placed at approximately $0.8 \mathrm{~m}$ from the cylinder rod at a polar angle of $90^{\circ}$ to measure the radiated noise. Noise measurements were first taken at free jet velocities of $20 \mathrm{~ms}^{-1}, 40 \mathrm{~ms}^{-1}$ and $60 \mathrm{~ms}^{-1}$, respectively, without the presence of the cylinder rod. It is expected that the dominant noise source in this case comes from the bare jet. After that, the cylinder rod was placed $100 \mathrm{~mm}$ downstream of the nozzle exit and the experiment was repeated at the three jet speeds as above. Note that the cylinder rod was held "freely" inside the potential core of the jet without the use of side plates. This means that the total length of the cylinder rod is larger than the width of the nozzle exit. The advantage of this configuration is that there is no contribution from the side plates, which are normally used to hold the test objects, to the radiated noise. However, impingement from the nozzle shear layer to the rod and some three-dimensional flow effects might also exist.

The noise results are summarized in Fig. 4. The tonal components associated with the cylinder rod follow a Strouhal numbers between $0.22-0.23$. This indicates that the tonal noise is likely to be produced by the vortex shedding behind the cylinder rod. The tonal components are shown to be well above the background noise produced by the bare jet, sometimes as large as $50 \mathrm{~dB}$ difference. At the jet speeds of $40 \mathrm{~ms}^{-1}$ and $60 \mathrm{~ms}^{-1}$, the broadband noise produced by the cylinder rod at frequencies above the tonal component is about $10 \mathrm{~dB}$ above the bare jet level. At a 
lower jet speed of $20 \mathrm{~ms}^{-1}$, and to some extents at $40 \mathrm{~ms}^{-1}$, the noise spectrum of the bare jet at the high frequency is similar to the noise floor level in the quiescent condition.

As a summary, all the tonal and broadband components produced by the rough surface cylinder rod are well above the background noise produced by the bare jet across the frequency range between $0.1-22 \mathrm{kHz}$.

(a)

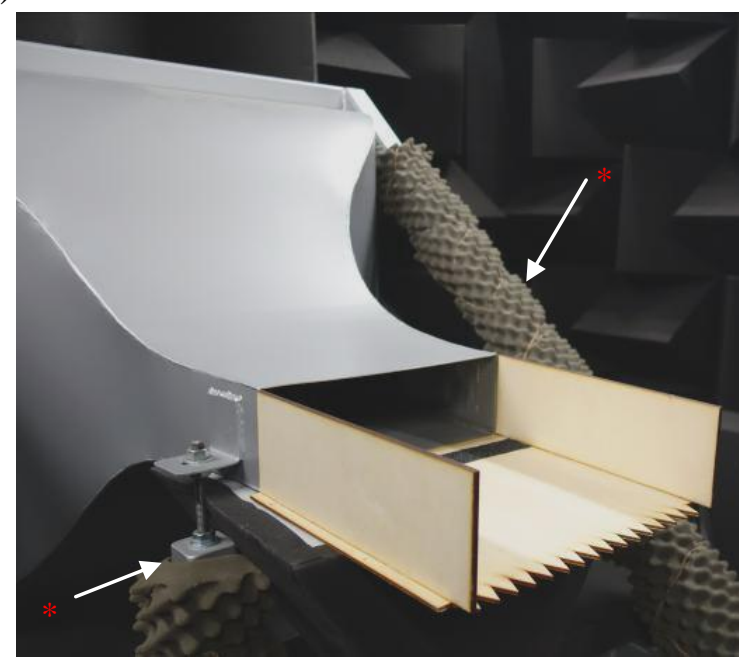

* - these struts have since been removed from the wind tunnel (b)

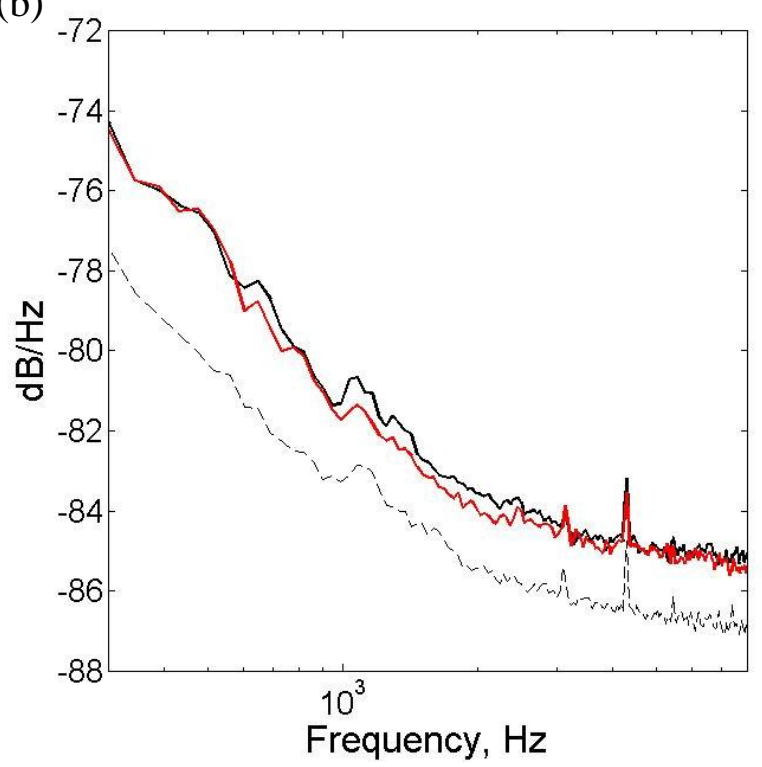

Figure 5. (a). Experimental setup for the measurements of self noise produced by flat plate fitted in turn with a straight trailing edge and a serrated trailing edges in a flat plate configuration; (b). Trailing edge self noise reduction at freestream velocity of $30 \mathrm{~ms}^{-1}$ : straight trailing edge (一); serrated sawtooth trailing edge ( - ) and the wind tunnel background noise produced by the bare jet (- - -).

\section{B. Flat plate broadband noise reduction by serrated trailing edge}

Contrary to the case (A) above, the test configuration chosen in this case represents a lower limit in terms of the measurable noise difference in aeroacoustics. The set up of the experiment is shown in Fig. 5a. A flat plate model of $300 \mathrm{~mm}$ (width) $\times 295 \mathrm{~mm}$ (length) was flush mounted to one side of the nozzle exit. Rough sandpaper was used to trip the flat plate boundary layer to turbulent near the nozzle exit. The trailing edge of the flat plate can be interchanged between a straight type and a serrated sawtooth type, which is defined by the following specifications: root-to-tip distance $(2 h)=20 \mathrm{~mm}$, and a serration angle $(\varphi)=25^{\circ}$.

Figure $5 \mathrm{~b}$ shows the far field noise measured over the flat plate fitted in turn with a straight trailing edge and a serrated sawtooth trailing edge. The microphone was placed at $0.8 \mathrm{~m}$ above the trailing edge at a polar angle of $90^{\circ}$. The jet speed was set at $30 \mathrm{~ms}^{-1}$. The noise spectral in Fig. $5 \mathrm{~b}$ demonstrates that the serrated sawtooth trailing edge produces a broadband noise level which is $0.5-1.0 \mathrm{~dB}$ lower than that produced by a straight trailing edge at a frequency range between $0.5-3 \mathrm{kHz}$. Several repeatability tests produce the same outcomes.

Some theories ${ }^{23,24}$ assume a full Kutta condition at the trailing edge when formulating expression for the far field noise. The current flat plate configuration means that flow is only present on one side of the surface, and there is no flow at the underside. The radiated trailing edge self noise level is therefore expected to be lower than when there is flow on both sides of the surface, such as in the airfoil case. This may explain the smaller amount of broadband noise reduction by a serrated sawtooth trailing edge in the flat plate configuration. Compared to an airfoil, broadband noise reduction between $3-5 \mathrm{~dB}$ can normally be achieved. This will be demonstrated in Fig. 6 in the next section.

As a summary, the background noise produced by the bare jet of the aeroacoustic wind tunnel is still considerably lower than the trailing edge self noise radiated under the current flat plate configuration, which allows quantification of the noise reduction when a serrated sawtooth trailing edge is used. 


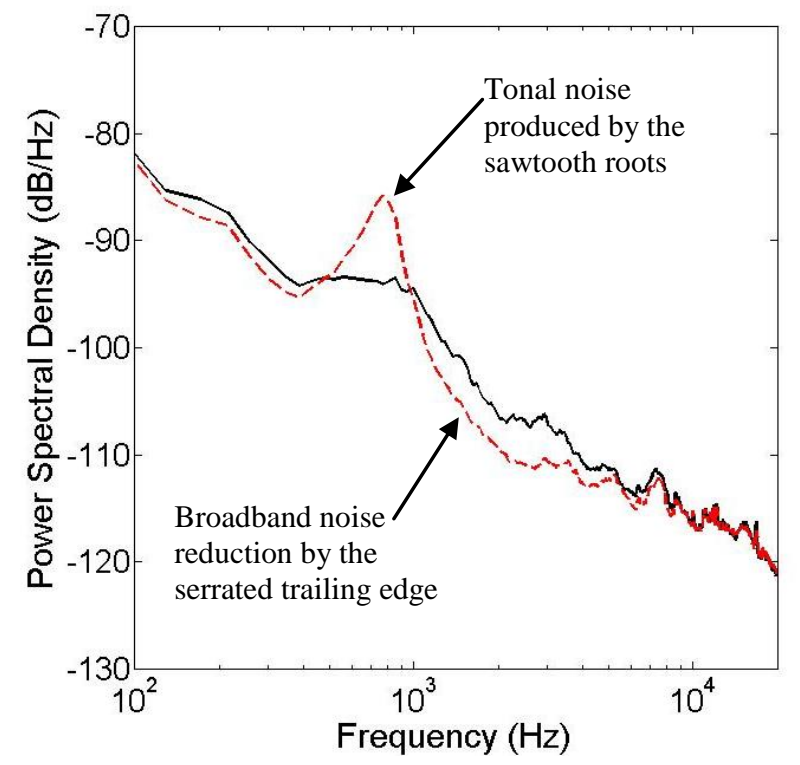

Figure 6. Comparison of the sound power spectral density $(\mathrm{dB} / \mathrm{Hz})$ for a NACA0012 airfoil with a straight trailing edge (-) and a nonflat plate type serrated trailing edge (- - -). The jet speed is at $25 \mathrm{~ms}^{-1}$ and the airfoil is set at zero degree angle of attack.

\section{Airfoil trailing edge broadband self noise}

One of the main studies which will be carried out in the anechoic wind tunnel in the future is the airfoil self noise. In this section, a preliminary test has been performed to measure the self noise of a NACA0012 airfoil with a straight trailing edge and a serrated sawtooth trailing edge (similar serration parameters as the flat plate case above). The airfoil has a chord length $(C)$ of $0.15 \mathrm{~m}$, and the width is similar to the width of the nozzle exit at $0.3 \mathrm{~m}$. The airfoil was fixed to the nozzle exit by two side plates. In this test the airfoil was set at zero degree angle of attack. Boundary layer tripping elements were applied to both sides of the airfoil at $x=0.1 C$ from the leading edge. The microphone was placed at about $1.1 \mathrm{~m}$ from the trailing edge at a polar angle of $90^{\circ}$. The free jet velocity was set at $25 \mathrm{~ms}^{-1}$.

Figure 6 shows the turbulent broadband noise spectra radiated by a straight trailing edge and a serrated trailing edge, respectively. Note that the serrated trailing edge is a nonflat plate type where a certain degree of bluntness exists at each sawtooth root. Vortex shedding has been shown to be emanated from the blunt roots ${ }^{9}$, which then proceeds to generate the tonal noise as depicted in Fig. 6. At frequency higher than the tonal component, between 1$7 \mathrm{kHz}$, significant broadband noise reduction has been achieved by the serrated trailing edge. Although not shown here, similar results can be obtained at higher velocity.

As a summary, the turbulent broadband noise results in Fig. 6 demonstrate that the aeroacoustic wind tunnel is suitable for airfoil self noise study. Other noise sources from the airfoil, such as the airfoil instability tonal noise, the blunt-induced tonal noise and the gust-leading edge interaction noise, are also quantifiable albeit the results are not shown here.

\section{Conclusion and outlook}

This paper represents the design principle of a quiet, low turbulence and moderately high speed aeroacoustic wind tunnel which was recently commissioned at Brunel University. A new hemi-anechoic chamber was purposely built to facilitate aeroacoustic measurements. Note that the design and characterization of the anechoic chamber are not covered in this paper. Given that both the wind tunnel and the anechoic chamber need to be built from scratch in an otherwise confined space, virtually every components are treated as the design "variables". For example, the final dimensions of the silencer plenum should depend upon the desired acoustical transmission loss, the contraction ratio of the nozzle, the preference of a small area ratio for the bend ducts, the size and location of the anechoic chamber and so on. Several design iterations are therefore needed to achieve the final layout. 
The free jet produced by the wind tunnel can achieve a maximum velocity of about $80 \mathrm{~ms}^{-1}$. The potential core of the free jet contains very low turbulence intensity $(0.1-0.2 \%)$. The noise characteristic of the aeroacoustic wind tunnel was validated by three case studies. All of which can demonstrate a very low background noise produced by the bare jet in comparison to the noises radiated from the cylinder rod/flat plate/airfoil in the air stream.

Due to the rather modest nozzle height at the exit, the aeroacoustic wind tunnel is only suitable for airfoil measurements at low to medium pressure loadings. For an airfoil at a large angle of attack, or a highly cambered airfoil is used, the jet deflection might be excessive. In addition, the nozzle shear layer could also be too close to the airfoil trailing edge. To overcome these, there is a plan to manufacture another nozzle with a larger exit area, conversely a reduced jet speed, in the future. To achieve a freeflow-like condition when the airfoil is set at a moderately large angle of attack, a method similar to the Virginia Tech's will be employed to connect the nozzle with a Kevlar-walled test section.

\section{Acknowledgment}

The constructions of the aeroacoustic wind tunnel and the hemi-anechoic chamber are financially supported by the School of Engineering and Design at Brunel University.

\section{References}

${ }^{1}$ Remillieux, M. C., Crede, E. D., Camargo, H. E., Burdisso, R. A., Devenport, W. J., Rasnick, M., Van Seeters, P. and Chou, A., "Calibration and demonstration of the new Virginia Tech Anechoic Wind Tunnel," AIAA Paper 2008-2911, 2008, 14 AIAA/CEAS Aeroacoustic conference and exhibit, Vancouver, Canada.

${ }^{2}$ Mathew, J., Bahr, C., Sheplak, M., Carrol, B. and Cattafesta, L. N., "Characterization of an anechoic wind tunnel facility," IMECE2005-81737, 2005, Orlando, Florida.

${ }^{3}$ Roger, M. and Moreau, S., "Broadband self-noise from loaded fan blades," AIAA Journal, Vol. 42, 2004, pp. 536-544.

${ }^{4}$ Moreau, S., Neal, D. and Foss, J., "Hot-wire measurements around a controlled diffusion airfoil in an open-jet anechoic wind tunnel," ASME: Journal of Fluids Engineering, Vol. 128, 2006, pp.699-706.

${ }^{5}$ Chong, T. P., Joseph, P. F. and Davies, P. O. A. L, "Design and performance of an open jet wind tunnel for aero-acoustic measurement," Applied Acoustics, Vol. 70, 2009, pp. 605-614.

${ }^{6}$ Gruber, M., Joseph, P. F. and Chong, T. P., "On the mechanism of serrated airfoil trailing edge noise reduction," AIAA Paper 2011-2781, 2011, 17 ${ }^{\text {th }}$ AIAA/CEAS Aeroacoustic conference and exhibit, Portland, Oregon.

${ }^{7}$ Polacsek, C., Reboul, G., Clair, V., Le Garrec, T. and Deniau, H., "Turbulence-airfoil interaction noise reduction using wavy leading edge: An experimental and numerical study," Inter-Noise, 2011, Osaka, Japan.

${ }^{8}$ Smith, M., Iglesias, E. L., Bremner, P. G. and Mendonca, F., "Validation test for flow induced excitation and noise radiation from a car window," AIAA Paper 2012-2201,18 ${ }^{\text {th }}$ AIAA/CEAS Aeroacoustic conference and exhibit, Colorado Springs, Colorado.

${ }^{9}$ Chong, T. P., Vathylakis, A., Joseph, P. F. and Gruber, M., "Self-noise produced by an airfoil with nonflat plate trailingedge serrations," AIAA Journal, Vol. 51, 2013, pp. 2665-2677.

${ }^{10}$ Sarradj, E., Fritzsche, C., Geyer, T. and Giesler, J., "Acoustic and aerodynamic design and characterization of a small-scale aeroacoustic wind tunnel," Applied Acoustics, Vol. 70, 2009, pp. 1073-1080.

${ }^{11}$ Geyer, T., Sarradj, E. and Fritzsche, C., "Measurement of the noise generation at the trailing edge of porous airfoils," Experiment in Fluids, Vol. 48, 2010, pp. 291-308.

${ }^{12}$ Geyer, T., Sarradj, E. and Fritzsche, C., "Silent Owl flight: Comparative acoustic wind tunnel measurements on prepared wings," Acta Acustica, Vol. 99, 2013, pp. 139-153.

${ }^{13}$ Leclercq, D., Doolan, C. and Reichl, J., "Development and validation of a small-scale anechoic wind tunnel," $14^{\text {th }}$ International Congress on Sound and Vibration, 2007, Cairns, Australia.

${ }^{14}$ Moreau, D., and Doolan, C., "Noise-reduction mechanism of a flat-plate serrated trailing edge," AIAA Journal, Vol. 51, 2013, pp. 2513-2522.

${ }^{15}$ Schumacher, K., Doolan., C. and Kelso, R. M., "The effect of a cavity on airfoil tones," Journal of Sound and Vibration, Vol. 333, 2014, pp. 1913-1931.

${ }^{16}$ Bilka, M., Anthoine, J. and Schram, C., "Design and evaluation of an aeroacoustic wind tunnel for measurement of axial flow fans," Journal of Acoustical Society of America, Vol. 130, 2011, pp. 3788-3796.

17 Ouyang, H., Zhu., Y. and Du, Z., "Design and characterization of a small aeroacoustic wind tunnel," Noise Control Engineering Journal, Vol. 58, 2010, pp. 67-73.

${ }^{18}$ Winkler, J., Temel, F. Z. and Carolus, T., "Concept, design and characterization of a small aeroacoustic wind tunnel facility with application to fan blade measurements," $3^{\text {rd }}$ International Symposium on Fan Noise, 2007, Lyon, France.

${ }^{19}$ Chong, T. P., Joseph, P. F. and Davies, P. O. A. L., "A parametric study of passive flow control for a short, high area ratio 90 degree curved diffuser,” ASME: Journal of Fluids Engineering, Vol. 130, 2008, pp. 111104-1-111105-12.

${ }^{20}$ Delany, M. E. and Bazley, E. N., "Acoustical properties of fibrous materials," Applied Acoustics, Vol. 3, 1970, pp. 105116. 
${ }^{21}$ Bradshaw, P., "Wind tunnel screens: flow instability and its effect on aerofoil boundary layers," Journal of Aeronautical Society, Vol. 68, 1964, pp. 168.

${ }^{22}$ Pinker, R. A. and Herbert, M. V., "Pressure loss associated with compressible flow through square-mesh wire gauzes," Journal of Mechanical Engineering Science, Vol. 9, 1967, pp. 11-23.

${ }^{23}$ Amiet, R., "Noise due to turbulent flow past a trailing edge," Journal of Sound and Vibration, Vol. 47, 1976 pp. $387-393$.

${ }^{24}$ Howe, M. S., "Trailing edge noise at low Mach numbers," Journal of Sound and Vibration, Vol. 225, 1999, pp. $211-238$. 